\title{
Proximal tibial pain in a child
}

\author{
Eric R. Henderson - Christina M. Jacobsen • \\ Rebecca A. Pohlmann • Harry P. W. Kozakewich • \\ Ingrid A. Holm • Mark C. Gebhardt
}

Published online: 27 February 2013

(C) ISS 2013

\begin{abstract}
Answer
Intraosseous, osteoblastoma-like phosphaturic mesenchymal tumor with secondary oncogenic rickets.
\end{abstract}

\section{Discussion}

Oncogenic osteomalacia is a rare condition in which mesenchymal-derived tumors secrete fibroblast growth factor 23 (FGF-23) [1]. The condition was described by McCance [2], although Prader discovered the tumoral etiology [3]. FGF-23 is normally secreted by osteocytes and osteoblasts and its action prevents both reabsorption of phosphorus in the renal tubules and the conversion of $25-\mathrm{OH}$ vitamin D to $1,25-\mathrm{OH}$ vitamin D. Elevated FGF-23 causes increased phosphate secretion resulting in rickets or osteomalacia $[4,5]$. A similar scenario to oncogenic osteomalacia

This investigation was performed at Boston Children's Hospital

The case presentation can be found at doi: 10.1007/s00256-013-1585-8

E. R. Henderson $(\bowtie)$

Department of Orthopaedic Surgery, Norris Cotton Cancer Center,

The Geisel School of Medicine at Dartmouth College,

One Medical Center Drive, Lebanon, NH, USA

e-mail: eric.r.henderson@gmail.com

C. M. Jacobsen · I. A. Holm

Divisions of Genetics and Endocrinology, Department of Pediatrics, Boston Children's Hospital, Harvard Medical School, Boston, MA, USA

R. A. Pohlmann • H. P. W. Kozakewich

Department of Pathology, Boston Children's Hospital,

Harvard Medical School, Boston, MA, USA

M. C. Gebhardt

Department of Orthopaedics, Beth Israel Deaconess Medical

Center, Harvard Medical School, Boston, MA, USA occurs in autosomal-dominant hypophosphatemic rickets in which gain-of-function mutations in FGF-23 lead to rickets and osteomalacia associated with low serum phosphorus levels [6].

The histopathological spectrum of phosphaturic mesenchymal tumor (PMT) is somewhat varied and, although fairly typical, does not readily lend itself to subclassification $[1,7]$. Two groups, however, have subdivided intraosseous PMT into four types. Named according to known tumors that they closely resemble, they are: mixed connective tissue, non-ossifying fibroma-like, ossifying fibroma-like, and osteoblastoma-like PMT [7]. Osteoblastoma-like PMT is an uncommon manifestation with four original cases described in the English, Pubmed-surveyed literature and two revisions of earlier diagnoses [1, 7]. These 6 patients were age 13 to 24 years, 3 were male and 3 were female.

Our patient's plain radiograph of the tibia demonstrated mild physeal widening consistent with rickets. A skeletal survey showed this finding to be consistent throughout the patient's skeleton (Fig. 1). The lesion itself was metaphyseal and lytic compared with the normal bone, but with a sclerotic and well-marginated border; it also demonstrated anterior cortical erosion. A differential diagnosis based upon radiographs alone would include non-ossifying fibroma, an early ossifying fibroma, and, less likely, chondromyxoid fibroma. This lesion demonstrates a narrow zone of transition, little or no periosteal reaction, no soft-tissue mass, and minimal cortical erosion, all of which suggest a nonaggressive histology [8]. Initial management of a patient presenting with these radiographs would be predicated on the lesion's benign characteristics. If the discovery of this lesion were purely incidental and our patient was without symptoms then observation would be a prudent course. A symptomatic lesion indicates that there is either structural weakness of the bone or the lesion is creating products that are chemically stimulating local nociceptors; either situation 
Fig. 1 a Anteroposterior and $\mathbf{b}$ lateral radiographs of the left wrist demonstrating widening of the physes consistent with the patient's diagnosis of rickets
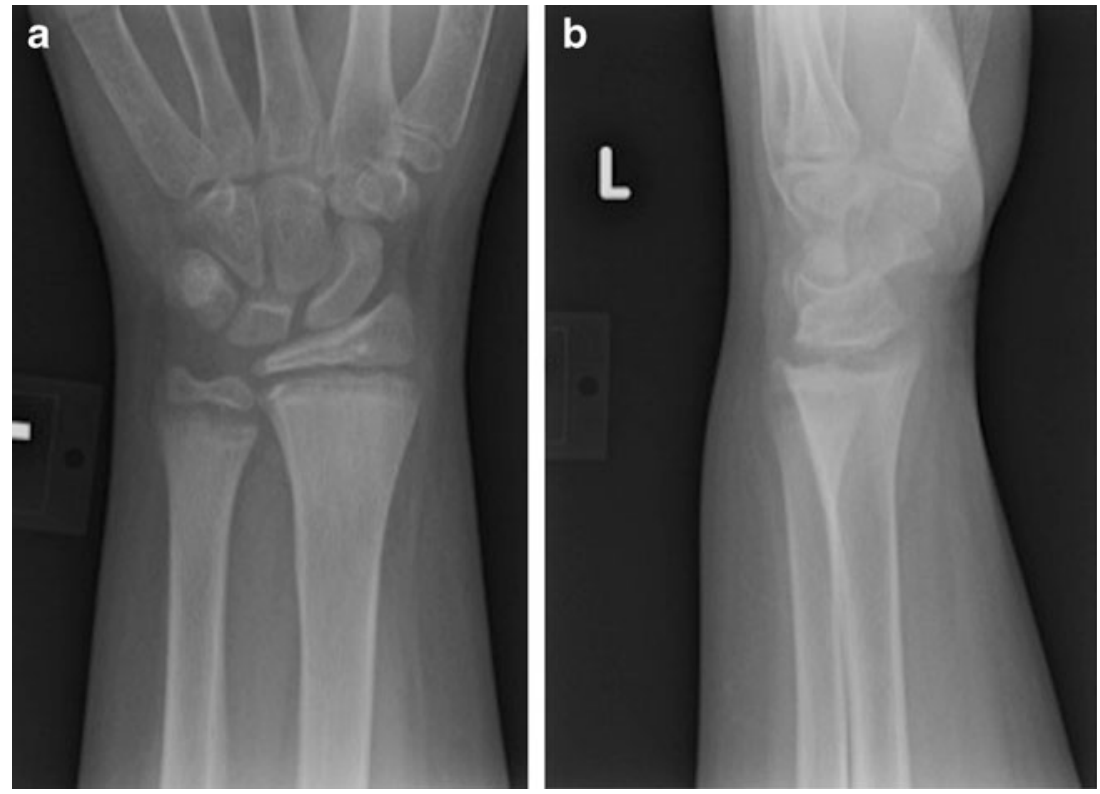

requires further inquiry into the lesion's nature in the form of imaging and biopsy.

Magnetic resonance imaging revealed cortical disruption with heterogeneous signal on T1- and T2-weighted images, no marrow edema on T2-weighted images, and minimal contrast uptake. In this case, MRI is not particularly helpful in narrowing the differential diagnosis as all of the aforementioned diagnoses can demonstrate mixed high- and low-signal images. The bone scan shows a modest increase in uptake at the lesion compared with normal bone. The needle biopsy submitted for consultation showed nodules and sheets of eosinophilic matrix, focally calcified and focally polarizable in a pattern similar to woven bone, as the dominant feature. Rather bland small spindle-shaped cells were present at the periphery of the matrix or as isolated cells or strands within it. Mitoses were rare and necrosis was absent. Occasional multinucleated giant cells were seen. The biopsy was interpreted as concerning for osteosarcoma, but because of the relatively bland histopathology and the clinical and imaging features that suggested an indolent tumor, a repeat needle biopsy was recommended, which revealed a similar histopathological appearance (Fig. 2). Additional consultation resulted in PMT being proffered as the most likely diagnosis with a suggested conservative but complete resection that might further help to clarify the nature of the tumor.

In our patient, dual-energy X-ray absorptiometry (DEXA) scanning confirmed the diagnosis of rickets with a whole-body bone mineral density of $0.64 \mathrm{~g} / \mathrm{cm}^{2}$, corresponding to a Z-score of $-3.8 \mathrm{SD}$. The initial FGF-23 level was $332 \mathrm{RU} / \mathrm{ml}$ (normal <230). Before resection of his tumor he was started on calcitriol $0.25 \mathrm{mcg}$ twice daily and phosphate $250 \mathrm{mg}$ four times daily to treat his bone pain and rickets. The tumor was resected en bloc via a hemicortical osteotomy followed by allograft and plate reconstruction (Fig. 3). Pathological examination of the resected specimen showed that the lesion was confined to the bone and that the margins of resection were free of tumor (Fig. 4). Histopathology was similar to that observed in the needle biopsies (Fig. 5). The final pathological diagnosis was phosphaturic mesenchymal tumor, osteoblastoma-like variant, which was

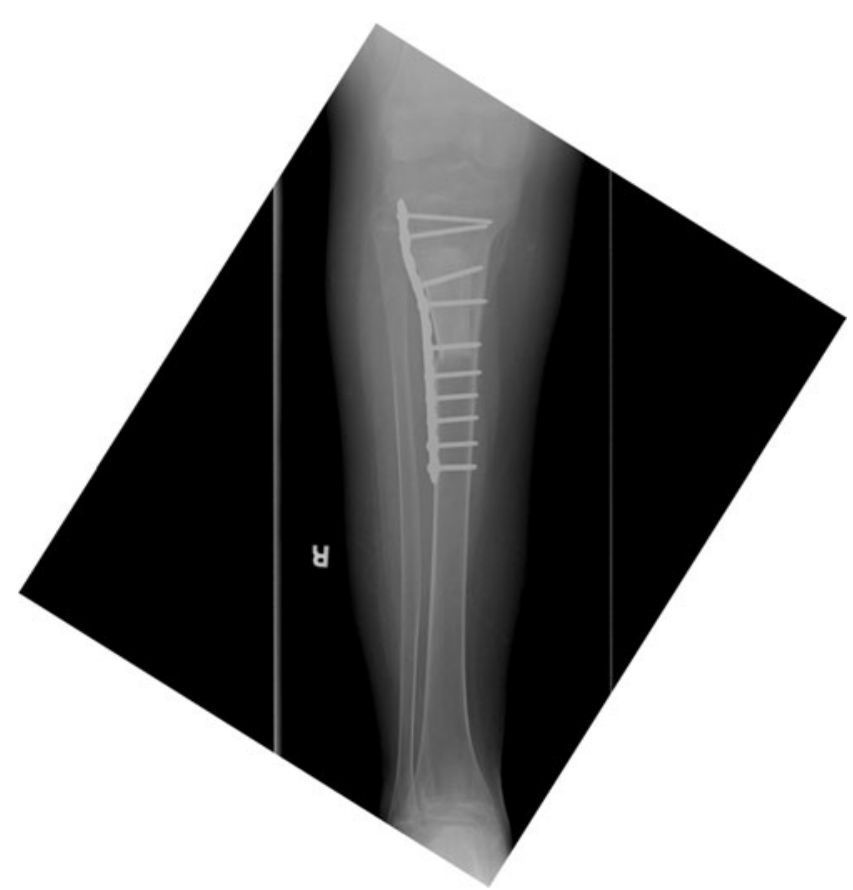

Fig. 2 Anteroposterior radiograph of the tibia 1 month after operation 

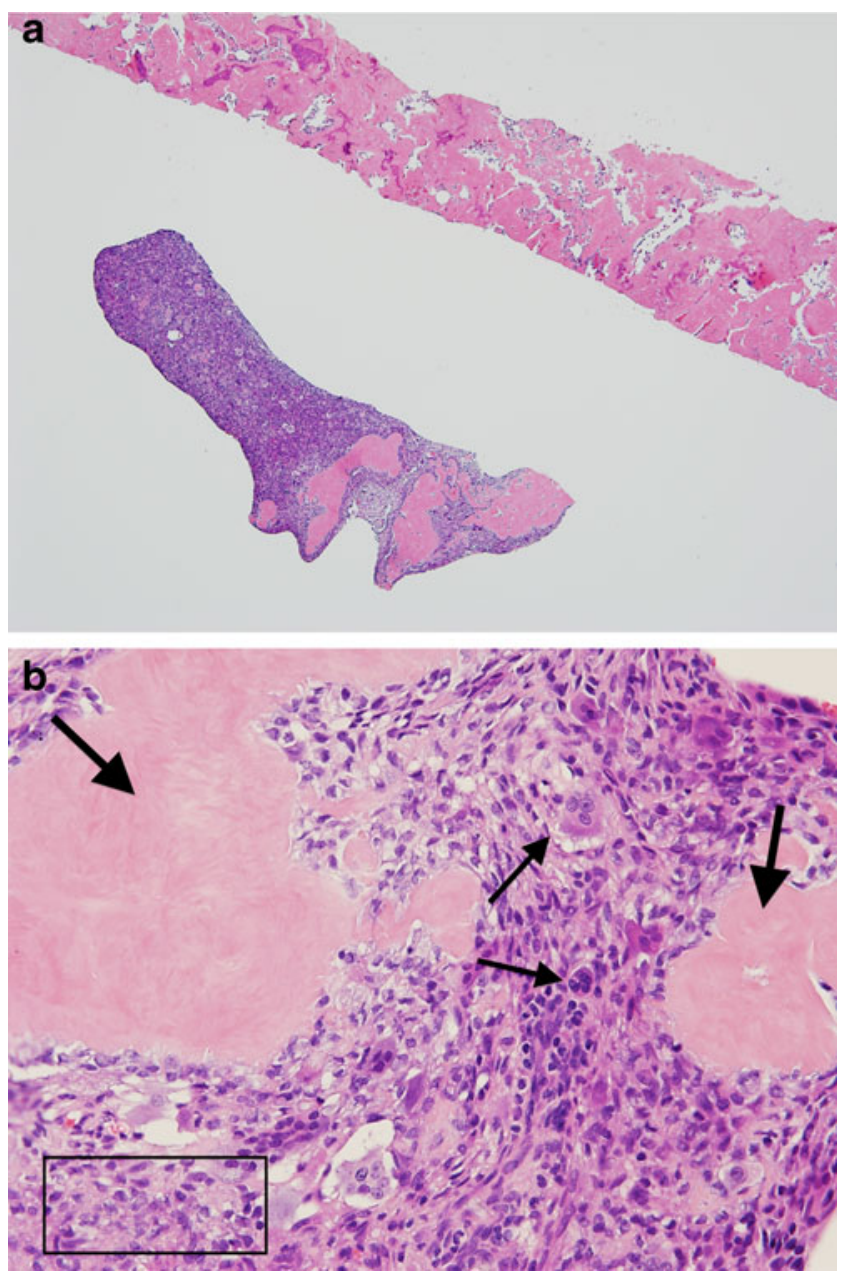

Fig. 3 a Low-power $(\times 40, \mathrm{H}$ and $\mathrm{E})$ histological image showing hypocellular, focally calcified osteoid-like matrix with interspersed cellular areas and b high-power $(\times 400, \mathrm{H}$ and $\mathrm{E})$ histological image showing small, spindle-shaped, bland lesional cells (box) combined with rare multinucleated giant cells (small arrows) surrounded by an osteoid-like matrix (large arrows)

histologically benign. Immediately following tumor excision, calcitriol and phosphate supplements were discontinued and he was started on calcium and vitamin D supplements. Two weeks after his operation the FGF23 level was $<50 \mathrm{RU} / \mathrm{ml}$.

One year following his operation the patient has no pain; hip, knee, and ankle range of motion are symmetrical to the contralateral side. He walks with a normal, plantigrade gait and participates in all activity except contact sports. His FGF-23 level remains $<50 \mathrm{RU} / \mathrm{ml}$.

Definitive treatment for oncogenic osteomalacia is surgical removal of the tumor, after which serum FGF-23 levels return to normal [9]. When resection is not feasible, patients may be managed pharmacologically. Treatment is identical to that for patients with hypophosphatemic rickets and includes phosphate and

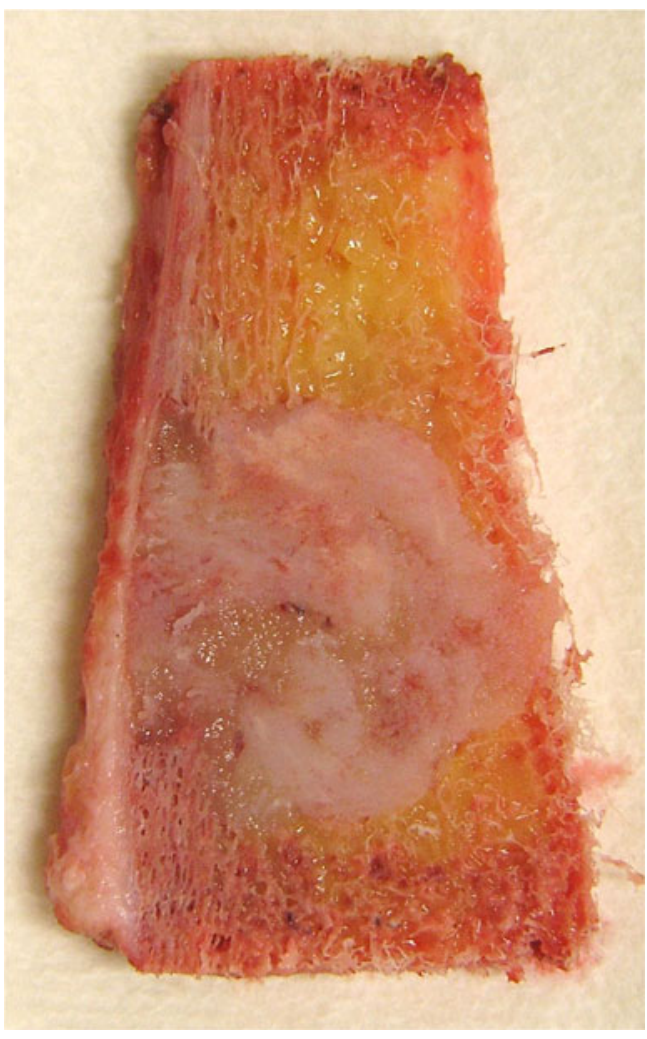

Fig. 4 Coronal section through a specimen resected in a hemicortical fashion with an irregular but well-marginated gray mass. The cortex at the zone of lesion abutment demonstrates cortical erosion (left aspect of the photograph)

1,25-OH vitamin D supplements [10]. After tumor resection, phosphate supplementation is discontinued; however, patients should continue calcium and 1-25$\mathrm{OH}$ vitamin D supplementation until bone density is restored.

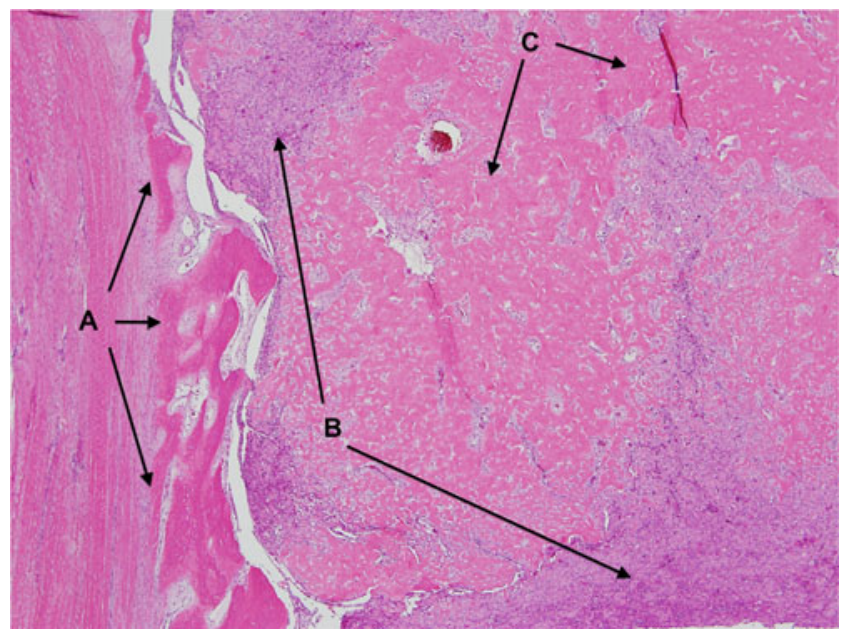

Fig. 5 The cortex is infiltrated and eroded, but not penetrated by the tumor $(A)$. Cellular areas $(B)$ representative of the initial biopsy specimens interspersed with extensive osteoid-like matrix $(C)(\times 40, \mathrm{H}$ and $\mathrm{E})$ 
Acknowledgements Appreciation is extended to Dr. Andrew Folpe at Mayo Clinic, Rochester, Minnesota, who evaluated the needle biopsies, detected expression of FGF23 in the tumor by RT-PCR, proffered a diagnosis of PMT and also reviewed the histopathology of the resected specimen.

Financial disclosures None of the authors has any financial disclosures.

\section{Conflicts of interest None.}

\section{References}

1. Folpe AL, Fanburg-Smith JC, Billings SD, Bisceglia M, Bertoni F, Cho JY, et al. Most osteomalacia-associated mesenchymal tumors are a single histopathologic entity: an analysis of 32 cases and a comprehensive review of the literature. Am J Surg Pathol. 2003;28(1):1-30.

2. McCance RA. Osteomalacia with Looser"s nodes (Milkman"s syndrome) due to a raised resistance to vitamin D acquired about the age of 15 years. Q J Med. 1947;16(61):33-46.
3. Prader A, Illig R, Uehlinger E, Stalder G. Rickets following bone tumor. Helv Paediatr Acta. 1959;14:554-65.

4. Perwad F, Portale AA. Vitamin D metabolism in the kidney: regulation by phosphorus and fibroblast growth factor 23 . Mol Cell Endocrinol. 2011;347(1-2):17-24.

5. Quarles LD. Skeletal secretion of FGF-23 regulates phosphate and vitamin D metabolism. Nat Rev Endocrinol. 2012;8(5):276-86.

6. White KE, Carn G, Lorenz-Depiereux B, Benet-Pages A, Strom TM, Econs MJ. Autosomal-dominant hypophosphatemic rickets (ADHR) mutations stabilize FGF-23. Kidney Int. 2001;60(6):2079-86.

7. Weidner N, Santa Cruz D. Phosphaturic mesenchymal tumors. A polymorphous group causing osteomalacia or rickets. Cancer. 1987;59(8):1442-54.

8. Lodwick GS, Wilson AJ, Farrell C, Virtama P, Dittrich F. Determining growth rates of focal lesions of bone from radiographs. Radiology. 1980;134(3):577-83.

9. Chong WH, Molinolo AA, Chen CC, Collins MT. Tumor-induced osteomalacia. Endocr Relat Cancer. 2011;18(3):R53-77.

10. Carpenter TO, Imel EA, Holm IA, Jan de Beur SM, Insogna KL. A clinician's guide to X-linked hypophosphatemia. J Bone Miner Res. 2011;26(7):1381-8. 